\title{
A Minimal Framework for Specification Theory
}

\author{
Bernd Baumgarten \\ German National Research Center for Information Technology (GMD) \\ Rheinstr. 75, D-64295 Darmstadt, Germany \\ baumgart@darmstadt.gmd.de \\ http://www.darmstadt.gmd.de/ baumgart/aspekte.html
}

\begin{abstract}
Notions concerning the specification, implementation, verification and testing of systems are ordinarily defined within a given context. We discuss what could and should belong to such a specification context. We obtain a unique practice-oriented specification semantics resp. conformance relation. We expect our framework to permit the definition of a general vocabulary for specification, verification and testing, independent of specific models or languages.
\end{abstract}

\section{Overview}

When dealing with specifications, we are usually doing so within some context. In the full paper we argue that a specification context should comprise some or all of the following components:

- a set Systs of systems of interest,

- a set $O b s$ of possible observations to be made of these systems,

- a set Props of potentially relevant system properties,

- a set Specs of specification terms (or specifications),

- a relation permits between systems and observations,

- a relation has_property between systems and properties, and

- a function obs_sem mapping each specification to a set of (permitted) observations.

In the full paper, we discuss in which regards these components form a useful framework covering more aspects than might be suspected at first sight, as well as special topics, for example why time is such an essential ingredient in observations.

Specification contexts have been used successfully

- to assess the significance of system requirements, and

- to give a formal meaning to formerly vague notions of testability.

We intend to use this framework to formalize a multitude of practically motivated concepts in testing (for a typical list, cf. ISO 9646 Part 1), as a contribution towards reliable and formally founded test generation procedures. We also plan to extend our concepts to aspects of concurrency, probability, and security.

For the full paper and related information, see the web page given above. 